\title{
Catastrophe Models and the Expansion Method: A Review of Issues and an Application to the Econometric Modeling of Economic Growth
}

\author{
EMILIO CASETTI \\ Department of Economics, Odense University, Odense, Denmark \\ (Received 15 May 1996; Revised 19 February 1997)
}

\begin{abstract}
Many negative reactions to Catastrophe Theory have been triggered by overly simplistic applications unintended and unsuited for statistical-econometric estimation, inference, and testing. In this paper it is argued that stochastic catastrophe models constructed using the Expansion Method hold the most promise to widen the acceptance of Catastrophe Theory by analytically oriented scholars in the social sciences and elsewhere. The paper presents a typology of catastrophe models, and demonstrates the construction and estimation of an econometric expanded cusp catastrophe model of economic growth.
\end{abstract}

Keywords: Catastrophe models, Expansion method, Economic growth

\section{INTRODUCTION}

Catastrophe Theory (CT), that originated with Thom's (1975) 'Structural Stability and Morphogenesis', aroused an initial intense interest that was later followed by a spate of criticisms. Today $\mathrm{CT}$ is very much alive, but perhaps is not having the impact it could and should. The major factors hampering its progress are (a) that many applications of CT are regarded as much too abstract and simplistic by substantive scholars, and (b) that CT has not entered yet to a sufficient extent into the modeling phase centered upon statistical econometric estimation and inference.

The focus of this paper is upon the application of CT. The paper discusses and implements a 'Modeling Perspective' intimating that the mathe- matical models of any aspect of reality are the central point of any application of mathematics. This perspective calls for defining what mathematical models are and how they are constructed. One of the two fundamental modes of model construction is by 'expansions', namely, by modeling the parameters of a preexisting model. The Expansion Method articulates the rationales and the operational specifics for constructing by expansions more complex and realistic models from simpler ones.

In the sections that follow, first the salient traits of CT are briefly reviewed. Then the Modeling Perspective and the Expansion Methodology are outlined and applied to catastrophe modeling, and in the process the scope and variety of catastrophe models is discussed and a typology 
of these models is proposed. Finally, to demonstrate the leitmotifs of the paper an expanded econometric cusp catastrophe model of modern economic growth is constructed and estimated.

\section{CATASTROPHE THEORY}

Let us begin from some generalities on the nature and significance of catastrophe theory. Over the years, the various components and themes of CT have been emphasized to different degrees. For instance, Thom's classification theorem had a prominent role early on, a lesser one in later years, and virtually disappears from consideration in the work of scholars such as Arnol'd. According to Arnol'd (1992, p. 2) "the origins of catastrophe theory lie in Whitney's theory of singularities of smooth mappings and Poicare and Andronov's theory of bifurcations of dynamical systems". It seems fair to say that together with Bifurcation Theory, Catastrophe Theory is today regarded as a branch of the modern Non-Linear Dynamics (Drazin, 1992; Tu, 1992; Glendinning, 1994).

It is to some extent a matter of interpretation what exactly CT is because of its evolution after its original statement by Thom. The literature on CT has been substantially influenced by early supporters such as Zeeman (1977) and Poston and Stewart (1978), by its critics (Zahler and Sussmann, 1977; Sussmann and Zahler, 1978; Arnol'd, 1992, p. 102 ff), and by the many scholars who used it and applied it in substantive fields. The reviews of CT applications such as the ones by Gilmore (1981), Wilson (1981), Lung (1988), Rosser (1991) and Guastello (1995), attest to the impact that CT has had.

A key point of CT since its very beginning is that 'systems' are found in 'stable equilibrium states'. Under conditions of 'structural stability' small changes in systemic 'control parameters' bring about small changes in these stable states. However, small changes in control parameters across 'critical' thresholds will cause stable equi- libria either to disappear, or to 'bifurcate' into multiple equilibria, some of which are stable. The appearance of multiple stable equilibria at critical points in control space is a special case of the bifurcations dealt with greater generality by $\mathrm{Bi}$ furcation Theory (Hale and Kocak, 1991).

In most early articulations of CT the stable equilibrium states are viewed as the optima of a function of the state variable(s), specifically, as the minima of a 'potential function'. The latter terminology follows from applications of CT in physics. However, CT has also a dynamic dimension the early development of which gained substantially from the work of Zeeman. 'Gradient' dynamic formulations corresponding to the ones based on the minimization of a potential function can be easily obtained by setting the time derivative(s) of the state variable(s) equal to minus the gradient of the potential function. This is equivalent to assuming that the state variable(s) will move downward on the potential manifold, following the direction of steepest descent and seeking the local minimum in the domain of attraction of which they are located. These 'gradient' equations link CT to the theory of nonlinear dynamics at large.

The minima of the potential function correspond to stable equilibria of 'gradient differential equation(s)'. At the critical point(s) in control space in correspondence to which local minima of the potential function disappear or multiply, in the phase diagrams of gradient systems stable equilibria disappear or multiply. This links CT to the qualitative analysis of non-linear differential equations that predates it by several decades.

Thom's mathematical contribution that motivated and started CT is the "classification theorem'. Essentially, Thom proved for 'seven elementary catastrophes' that for a wide class of functions, in the neighborhood of their 'degenerate singularities', the types of catastrophes that can occur are the same that characterize the corresponding canonical catastrophe equations. For example, a broad class of functions involving one state variable and two control parameters will 
have near a degenerate singularity either a fold or a cusp catastrophe, same as the canonical catastrophe equation with one state variable and two control parameters.

This brief articulation of the major points of CT begs the question of whether there are distinctive contributions that should be credited to $\mathrm{CT}$, and on which, ultimately, the applications of CT can rest.

Its critics deny that $\mathrm{CT}$ represents a distinctive contribution, or at least a substantial one. They contend that the classification theorem is only valid 'locally', in the neighborhood of degenerate singularities, and consequently does not justify attributing a generality of scope to the elementary catastrophes. Also, they would argue that the discontinuities in the elementary catastrophes are not unique to CT, but can be produced within Bifurcation and Non-Linear Dynamics theories as special cases. Furthermore, they have contended that the examples of applications of CT are contrived, and better models with same effects can be put together outside CT.

However, it can be argued that CT has been having an important role along at least three dimensions. Namely, (a) it has etched into the collective consciousness of the scholars interested in modeling dynamic phenomena that systemic equilibria may appear disappear or multiply when control parameters move across critical thresholds; (b) it has identified 'types' of catastrophes, some of which have been found very useful in many applications; and (c) it has linked the appearance, disappearance, and bifurcation of equilibrium states simultaneously to dynamic formalizations and to formalizations in terms of systemic optima. Such twin formalizations are synergistic in their potential to inspire substantive theory and empirical analyses.

Let us comment briefly only on the first of these three points. Before CT, a widespread collective awareness that discontinuities can follow the smooth change of systemic parameters across critical thresholds did not exist; after CT it did. It can be argued that the possibility of having multiple stable equilibria, as well as the possibility for a system to switch across topologies characterized by different constellations of such equilibria as a result of changes in systemic parameters, fall within the scope of non-linear dynamics, as a subset of the state variables' behaviors considered within it. It could be also argued that in the literature there are applications along these and similar lines that predate CT or were developed independently from it. For instance the critical minimum effort thesis (Oshima, 1959; Leibenstein, 1957), and Nelson's (1960, 1965) contribution to the modeling of the escape from the Malthusian trap were related to the qualitative analysis of differential equations but can be easily viewed in terms of CT.

These and similar examples notwithstanding, it is to the credit of CT's originator and of the many scholars who have contributed to CT over the years, to have generated a collective consciuousnes of catastrophes and catastrophe modeling. The notion that qualitative jumps across topologies may result from the continuous change of control parameters across critical thresholds may very well have been implicit in other scholarly traditions. Yet it is primarily due to CT if today, in the investigation of social biological and physical phenomena, continuous change as well as discontinuous qualitative change represent alternatives to be both considered and modeled.

\section{MATHEMATICAL MODELING}

All applications of mathematics, including those of catastrophe theory, start from, and/or are based upon, mathematical models of realities. The mathematical models of some phase of reality are not just a particular type of models (a 'species' within a 'genus' encompassing all types of models). Rather, they are conceptual artifacts qualitatively distinct from anything else called 'models'. A recognition of this distinctiveness, and of the fact that any application of mathematics is via mathematical models, are necessary 
prerequisites for understanding the roles of mathematical models, and the nature of the boundary that separates and links mathematics to its applications. Here, this 'Modeling Perspective' is briefly articulated in generalities, and subsequently is applied to catastrophe modeling. In this section we focus upon the definition of mathematical models and of the major approaches to their construction.

\section{Definition}

The mathematical modeling of some phase of reality ('mathematical modeling' for short), involves the linking of a substantive conceptual frame of reference to analytical mathematical structures. Mathematics defines analytical structures such as equations, inequalities, probability distributions, stochastic processes and so on, in which variables, random variables, and parameters appear. The substantive scientific disciplines concerned with the study of any aspects of reality, in the social sciences and elsewhere, define entities ('objects') with which they are concerned, variables that take specific values for these objects, and relations among these variables. The mathematical models of any realities consist of analytical structures with some or all of the variables, variates, and parameters in them linked to a substantive conceptual frame of reference, namely, to substantive variables and variates. The mathematical models can be deterministic, stochastic, or mixed. There are two major approaches to the construction of mathematical models: the conventional modeling and the expansion modeling.

\section{Conventional Modeling}

We can recognize at least two types of conventional model construction, which will be referred to as $\mathrm{A}$ and $\mathrm{B}$. The type $\mathrm{A}$ consists in the straightforward linking of an analytical structure to a substantive frame of reference. The type B involves extracting a new mathematical model from preexisting one(s) by a variety of mathematical manipulations. As example of type A model construction suppose that a substantive discipline has defined variables and important relations among these variables. A scholar from this discipline who has in his/her mind an inventory of analytical structures, selects one of these structures and links it to one such important relation. When this link is established, a mathematical model of some aspect of reality is born.

The conventional model building of type B consists in extracting models from other models by mathematical manipulations such as 'solving' or 'optimizing'. Suppose for example that we obtain a demand function by maximizing a utility function subject to a budget constraint. The demand function is a mathematical model, but so are also the utility function and the budget constraint. Another example is solving a differential equation that relates the rate of change of a country's population to its population size. The solution of the differential equation and the differential equation itself are both mathematical models.

It is important to note that the type B conventional modeling can be viewed in distinct but equivalent ways, depending upon whether the mathematical manipulation(s) it involves are carried out on a mathematical structure or upon a model. Consider the differential equation relating rates of change and levels of population. Here the mathematical manipulation involved consists in solving a differential equation. If the differential equation is a model because a substantive (demographic) frame of reference has been linked to it, then solving the differential equation yields a second model that, we can say, has been created by a type B conventional modeling. If instead the differential equation, taken as a mathematical structure, is solved to produce a second mathematical structure that is then linked to a demographic frame of reference, the resulting model is produced by a conventional modeling of type $\mathrm{A}$. 


\section{Expansion Modeling}

The expansion modeling (Casetti, 1972, 1986, 1997) consists in the conventional modeling of a preexisting ('initial') model's parameters, and usually involves the following:

(a) An 'initial model' is specified. This model may involve variables and/or variates, and at least some of its parameters are in letter form.

(b) Some or all of the letter parameters of the initial model are modeled by redefining them into functions of expansion variables or variates by 'expansion equations'. The expansion variables identify substantively relevant dimensions in terms of which the initial model's drifts, and the expansion equations are a specification of this drift.

(c) The initial model and the expansion equations constitute a 'terminal' model in structural form. If the right-hand sides of the expansion equations are substituted for the corresponding parameters in the initial model a reduced form 'terminal' model is obtained. A terminal model in either structural or reduced form encompasses simultaneously the initial model and a specification of its parametric drift across the space spanned by the expansion variables.

(d) This process can be iterated, with the terminal model produced by a previous cycle becoming the initial model of the next.

Since the expansion modeling involves the conventional modeling of an initial model's parameters that can be of types $A$ or $B$, also the expansions can be of types A or B. In type A cases an expansion equation results from the linking of substantive variables (some of which are the parameters of the initial models redefined as variables) to a suitable analytical structure. Instead, in a type B situation the expansion equations are arrived at via multiple steps. To exemplify, suppose that the parameters $\alpha$ and $\beta$ of a model $y=\alpha+\beta x$ are expanded by the differential equations (DEs) $\mathrm{d} \alpha / \mathrm{d} t=f(z)$ and $\mathrm{d} \beta / \mathrm{d} t=g(z)$. Both these DEs and their solutions qualify as expansion equations. However, if the solutions of the DEs are selected as expansion equations, clearly they are arrived at by an intermediate manipulation that in this case consists in a 'solving'. In passing, let us note that in this particular example the parameters appearing in the initial conditions from solving the DE can be also expanded.

The redefinition of an initial model's parameters into functions of expansion variables can be implemented in two equivalent ways, depending upon whether an analytical mathematical structure or a model are expanded. We can start from a mathematical structure, expand some or all the parameters in it, thus generating an expanded structure, and then link a substantive frame of reference to this expanded structure. Alternatively, we can start from a mathematical model and expand some or all of its parameters into functions of expansion variables. Both of these qualify as expansion modeling, and yield the same terminal model. In both cases the terminal model arrived at consists of an initial model complemented by models relating some or all of its parameters to expansion variables or variates.

Most models and analytical mathematical structures can be conceptualized as resulting from previous expansion(s), which in itself opens vistas useful to interpret existing models, and to view them in terms of a unifying perspective. However, any models or structures can be also regarded as the potential building blocks of more complex 'expanded' models and structures. This latter perspective proves especially useful when the models or structures so viewed possess a distinctive identity in the literature and in the consciousness of communities of scholars. In fact, this perspective provides one of the motivations for defining classes of 'initial' catastrophe structures or models and of more complex structures or models generated from these by expansions.

Several rationales of the expansion modeling are discussed in Casetti (1997). Here let it suffice 
to mention the model context rationale, which suggests that models can be related by expansions to external environments or contexts defined by contextual variables. If a model's parameters are expanded into these contextual variables, the terminal model obtained encompasses both the initial model and a specification of its drift across the context. There is a very broad range of situations to which this model context frame of reference can be applied.

\section{CATASTROPHE MODELS}

Mathematical models were defined as the resultant of linking analytical mathematical structures to substantive frames of reference. Let us now consider which types of models have arisen or can arise from linking catastrophe structures to substantive theory. Typologies of catastrophe models can be based on the analytical structures involved, on the substantive frames of reference attached to these structures, on the manner in which these linkages are established, and finally on the roles that these models have played or can play in the conduct of enquiry and in modeling literatures.

Let us focus here on typologies based on analytical catastrophe structures, that apply also to the models in which these structures appear. In the process, we will touch upon the possible relation of supportive or critical reactions to CT that can be traced to the comparative abundance or scarcity in the catastrophe literature of certain types of models/structures.

The following typologies will be discussed: (a) the elementary catastrophe models and their duals; (b) gradient and potential models; (c) canonical, generalized canonical, and non-canonical models; (d) expanded and non-expanded models; (e) deterministic and stochastic models.

These typologies are concurrent, so that a catastrophe model or structure can be classified in terms of all the five dimensions outlined above. Thus, for instance, we can have a dual-cusp canonical unexpanded deterministic gradient catastrophe model. It is useful to note that a large number of different catastrophe models exist or can be constructed. It is not necessarily true that all catastrophe scholars are aware of all of them. The scope and usefulness of applied catastrophe work can benefit from a greater awareness of the many options available. Let us now proceed with a discussion of these typologies taken in sequence.

\section{Primal and Dual Catastrophe Models}

In this paper we will confine ourselves to the 'elementary catastrophes', which are the fold, cusp, swallowtail, butterfly, plus the hyperbolic elliptic and parabolic umbilics. The first four involve one state variable, the umbilics, two. The identification of specific types of catastrophes and of their 'duals' (Wilson, 1981, p. 28; Poston and Stewart, 1978, p. $116 \mathrm{ff}$ ) is a major contribution. It goes beyond the mere recognition that 'qualitative changes' in dynamics, for instance in differential equations, may be produced by the continuous change of some parameters across critical thresholds. The elementary catastrophes represent specific types of qualitative change, some of which have been found very useful to understand many diverse social biological and physical phenomena.

To clarify, consider the cusp catastrophe, which in this paper is singled out for use in every example and in the demonstration. The cusp catastrophe and its dual involve one state variable and two control parameters, and corresponds to a well-specified constellation of stable and unstable equilibria. The cusp catastrophe includes a topology with a single stable equilibrium, and a topology with a low and a high stable equilibria and an intermediate unstable one. For suitable smooth changes in parameters a cusp catastrophe structure/model can switch from a single low level stable equilibrium condition, to a condition characterized by two coexisting stable equilibria, one low and one high, to a subsequent condition with a single high level stable equilibrium, or vice versa. 
The dual cusp catastrophe (Gilmore, 1981, p. 267-270) includes a topology with a single unstable equilibrium, and a topology with a low and a high unstable equilibria with an intermediate stable one. Smooth changes in parameters can produce sequential transitions from a single low level unstable equilibrium, to a condition characterized by an intermediate stable equilibrium positioned between two unstable ones, to a condition with a single unstable equilibrium, and backward. In a dual cusp catastrophy (as in any dual catastrophe) the equilibria occur at the same values of the state variable that yield equilibria in the primal, however what are stable equilibria in the primal become unstable in the dual, and vice versa. The primal cusp models (but not the dual cusp models) proved very useful in a great many fields and applications.

\section{Potential and Gradient Models}

Each primal and dual catastrophy can be formalized by potential or gradient structures. To exemplify, the canonical potential structures of the primal and dual cusps are

$$
F_{\mathrm{P}}(x)=\frac{1}{4} x^{4}+\frac{1}{2} u x^{2}+v x
$$

and

$$
F_{\mathrm{D}}(x)=-\left(\frac{1}{4} x^{4}+\frac{1}{2} u x^{2}+v x\right),
$$

where the subscripts P and D stand, respectively, for primal and dual.

The canonical gradient cusp structures are

$$
\dot{x}=-\left(x^{3}+u x+v\right)
$$

for the primal cusp and

$$
\dot{x}=x^{3}+u x+v
$$

for the dual cusp, where $\dot{x}$ denotes the derivative of $x$ with respect to time.
In general, the relations between primal and dual catastrophes are

$$
F_{\mathrm{D}}=-F_{\mathrm{P}}
$$

and the relations between potential and gradient formulations are

$$
\begin{gathered}
\dot{x}=-\operatorname{grad}(F), \\
F=-\int \dot{x} \mathrm{~d} t .
\end{gathered}
$$

These analytical structures become mathematical models when the variables and parameters in them are linked to a substantive frame of reference. Since these typologies differentiate types of analytical mathematical structures, they are also typologies of catastrophe models.

That a catastrophe can be formalized using either potential or gradient structures is a strong point of catastrophe modeling. Within the frame of reference of CT the systemic equilibria correspond to the optima of a potential function, that are also the stable equilibria of the gradient differential equations implied by the potential function. These twin formalizations open the way to developing substantive theory in terms of both systemic optima and their related dynamics (Casetti, 1991). This possibility, however, does not appear to have received as much attention as it deserves.

\section{Canonical and Non-canonical Models}

Examples of the canonical analytical catastrophe structures are the cusp potential equation (1) and the cusp gradient equation (3). All the canonical equations of the elementary catastrophes (cf. for instance Wilson, 1981, p. 29) are characterized on their right-hand sides by polynomials with some parameters set at numerical values and some terms missing. In fact, the missing terms can be regarded as having parameters set to a value of zero. The letter parameters in the canonical equations are the 'control parameters' that determine 
the topology of the equation. Let us call 'generalized canonical equations' the equations obtained by replacing the polynomials in the canonical structures by polynomials of the same degree but with all the parameters in letter form.

As an example, the generalized gradient canonical equation for the cusp is

$$
\dot{z}=\alpha_{3} z^{3}+\alpha_{2} z^{2}+\alpha_{1} z+\alpha_{0} .
$$

It should be noted that Eq. (8) encompasses the primal and the dual cusp canonical equations as special cases.

In order to link the $\alpha$ 's of the generalized canonical equation (8) to the control parameters $u$ and $v$ of the canonical equation (3) let us procede in two steps, as follows. First, let us partially generalize the canonical equations so that it will encompass the primal and dual cusps as special cases. To this effect, write

$$
\dot{x}=h\left(x^{3}+u x+v\right) .
$$

For $h=-1,1$ Eq. (9) specializes to the primal or dual canonical gradient cusp equation. The second step defines the shift transformation

$$
z=x-w
$$

which leads to

$$
\dot{z}=h\left((z+w)^{3}+u(z+w)+v\right) .
$$

Equation (11) defines a link between the $\alpha$ 's of the generalized equation (8) and the control parameters of the primal and dual cusp canonical equations (3) and (4). This link will be revisited and elaborated upon later in this paper. The $\alpha$ 's can be regarded as reduced form parameters, while the link parameters $h$ and $w$ plus the control parameters $u$ and $v$ will be referred to as structural parameters of the generalized cusp catastrophe equation. Alternative approaches to generalizing canonical models are discussed for instance in Brown (1995, p. 61) and in Cobb and Zacks (1985, p. 798).
A difference between generalized canonical and canonical models is in that the former influence through $h$ the speed at which the state variable(s) approach their stable equilibria. This is in contrast with conventional CT, which presupposes that systems are in stable equilibrium states.

The perfect delay and the Maxwell conventions are rules for determining in which equilibrium states the system is found when multiple equilibria materialize. According to the first convention the system remains in an equilibrium state until its disappearance. In terms of the Maxwell convention the system jumps from an existing equilibrium state to a better one as soon as the better equilibrium appears or becomes better. Both conventions, however, presuppose that the system instantly reaches an equilibrium and follows it as it changes.

In the generalized canonical gradient models, when the values of the control parameters and of the shift parameter $w$ are fixed, the speed at which stable equilibria are approached is determined by the parameter $h$. Thus, $h$ identifies a measurable systemic attribute.

The non-canonical catastrophe models are any 'other' models (namely, neither canonical nor generalized canonical) possessing the topologies that characterize any given catastrophe (cf. for example Wilson and Kirby 1980 p. 344 ff).

\section{Expanded and Non-Expanded Models}

In order to clarify the significance and importance of this typology let us start by applying it to 'canonical' models. For each $n$-tuplets of control parameter values, a canonical catastrophe structure or model corresponds to a specific topology characterized by a constellation of one or more stable equilibria with an appropriate complement of unstable equilibria. It does not matter whether these models are of the gradient or potential type, and to which elementary catastrophe they correspond: for one set of parameters they will all correspond to one specific constellation of 
stable and unstable equilibria. In order to be able to express the transition across topologies and/or the change of their equilibrium values across 'contexts' these parameters have to change. In keeping with the 'modeling' section of this paper, we can formalize this change by expanding the control parameters into functions of other variables.

A large number of expansions are possible. Here, let us focus upon expansions with respect to time, and with respect to one or more variables indexing some appropriate context of the model other than time. To clarify the rationale and usefulness of the expanded catastrophe models, consider a model that for different values of its parameters is characterized by one of the topologies typical of a particular catastrophe. In itself this model is suited for 'comparative statics' analyses, but unsuited to model a switch in topologies over time, or across a 'context'. However, if this model's parameters are expanded with respect to time or with respect to substantive contextual variables, the resulting terminal model can portray and resolve a switch in topologies over time or across the substantive context.

In the case of the gradient cusp catastrophe model (3) a duplet of numerical values of $u$ and $v$ corresponds to a specific cusp topology. Consequently, if $u$ and $v$ are estimated from empirical data we can determine the topology implied by the data. A comparative statics analysis involves comparing the topologies corresponding to alternative data sets. However, a transition across topologies is outside the scope of this model. Instead, if $u$ and $v$ are redefined into functions of time $t$,

$$
\begin{aligned}
& u=c_{\mathrm{U} 0}+c_{\mathrm{U} 1} t+c_{\mathrm{U} 2} t^{2}+\cdots, \\
& v=c_{\mathrm{V} 0}+c_{\mathrm{V} 1} t+c_{\mathrm{V} 2} t^{2}+\cdots,
\end{aligned}
$$

the terminal model obtained by replacing the $u$ and $v$ in (3) with the right-hand sides of (12) and (13), for appropriate values of the $c_{\mathrm{U}}$ and $c_{\mathrm{V}}$ parameters, can produce a switch across the cusp catastrophe topologies over time.
The catastrophe literature on the fast and slow dynamics deals with an interesting class of expansions of canonical catastrophe models. This literature differentiates between the dynamics of the fast variables (the state variables), and the dynamics of the slow variables that span the control space. The slow dynamics is formalized by differential equations which specify the rate of change over time of control parameters such as $u$ and $v$ in the example above, as a function of time, of control parameter 'levels', or of slow variables. The fast variables adjust rapidly to their stable equilibrium levels, so that a system characterized by a fast and slow dynamics will reflect the changes in the stable equilibria of the fast variables resulting from the changes in control parameters produced by the slow dynamics. An early example is given in Zeeman (1972, 1973). In the fast/slow dynamics formalisms, the slow-dynamics equations are expansion equations of the initial model's (control) parameters, while the initial model is represented by the fast variables' equations.

The differential handling of the fast and slow variables constitutes an important methodological contribution implicit in Thom's initial formulation of the catastrophe theory (Thom, 1975), but made explicit and placed into focus by Zeeman (1977, p. 65 ff). Its use in connection with the application of non-linear dynamics in the spatial sciences has been advocated and theorized by Dendrinos and Mullally (1981, 1985) and Dendrinos and Sonis (1990). A related frame of reference in which fast and slow dynamics concepts appear is Haken's 'synergetics'. Haken (1983) views dynamic systems in terms of slowly moving 'order parameters' and fast moving 'slave' variables or subsystems.

In the catastrophe and non-linear dynamics literatures, we encounter variables that are fast, variables that are slow, and constants. Also, the fast-slow dychotomy itself may be replaced by multiple 'relative speeds of change' (Wilson, 1981; Dendrinos, 1989). A number of studies modeling multilevel time scales are reviewed in 
Rosser (1991, p. 212). These multilevel time scales can be conceptualized as involving iterated expansions.

In the expanded canonical catastrophe models discussed in the previous paragraphs it is one or more control parameters that are expanded. If we expand the generalized canonical models, expansions can be carried out on the control parameters and/or on the link parameters such as $h$ and $w$ in the case of the cusp catastrophe (Eq. (11)). Suppose that only $h$ and $w$ in (11) are expanded, say, with respect to time. The resulting terminal model cannot display a catastrophic switch in topology, but is instead capable of accomodating temporal shifts in the values of the state variable corresponding to stable or unstable equilibria via changes in $w$, and a transition from a catastrophe to its dual via shifts in $h$ carrying this parameter through a change in sign. If also $u$ and $v$ are expanded, the resulting terminal model can also accomodate catastrophic changes in topology.

The expansion of a generalized canonical model can be carried out with respect to structural parameters such as $h, w, u$, and $v$ for the cusp, but also with respect to the reduced form parameters such as the $\alpha^{\prime}$ s in (8). If the latter are expanded, at each point in expansion space a set of $\alpha$ 's becomes defined from which the values of the structural parameters for that point can be computed. This is the approach applied in the demonstration presented later in this paper. The implications of expanding some or all the parameters of a non-canonical catastrophe model are likely to be model specific and no attempt is made here to address them in generalities.

\section{Deterministic and Stochastic Models}

With some notable exceptions such as for instance Guastello (1982, 1987, 1988), CT has not entered yet to a sufficient degree into the inferential stage, and tends to be identified with abstract deterministic models by scholars from fields in which preferences for models intended for inference are firmly established.
Yet, though, catastrophe models and structures can be deterministic or stochastic. The deterministic models are useful to formalize theory and to identify modalities of phenomena, but cannot be used for validation based on estimation and inference. For these, the deterministic models have to be converted into stochastic models by reformulating them as stochastic processes and/or by adding error terms to them.

The stochastic models can be differentiated into statistical and econometric, although the difference between these is not clear cut. The statistical models tend to be constructed by reformulating a deterministic catastrophe structure as a stochastic non-linear difference or differential equation. These equations can be investigated analytically, numerically, or by simulations, to obtain the probability density functions of the state variables that they imply, and in order to determine appropriate estimation approaches (Cobb, 1978; 1992; Cobb et al., 1983; Cobb and Zacks, 1985).

The econometric catastrophe models can be constructed by adding error terms to deterministic models while at the same time redefining some or all of their variables into random variables. Econometric modeling is employed in the example discussed later in this paper. First, though, let us touch upon some aspects and themes of econometrics that are relevant to catastrophe modeling.

A major portion of econometrics centers on the estimation based on empirical data of deterministic relationships that originated in mathematical economics. In fact, some authors have identified econometrics with this tradition. For example Johnston (1963, p. 3) writes "Economic theory consists of the study of various groups or sets of relations which are supposed to describe the functioning of a part or the whole of an economic system. The task of econometric work is to estimate these relationships statistically...". At p. 4 he adds "...for measurement and testing purposes, [deterministic] formulations ... are inadequate. The extension employed is the 
introduction of a stochastic term into economic relationships." While econometrics had its origin with the conversion of the deterministic models from mathematical economics into stochastic models suited for estimation and inference, over time its scope has become much wider. Any deterministic relation with theoretical foundations not from economics, or without substantive theoretical foundations, can be converted into econometric models by the addition of error terms. Consequently, also deterministic catastrophe models can be transformed into econometric formulations.

In the earlier econometrics the error terms were assumed to be well behaved RVs, normally and independently distributed and with expectation zero and identical variances. Today, however, the assumptions of independence (temporal and spatial) and of homoschedasticity are routinely tested, and when the null hypotheses of independence and homoschedasticity are rejected the conversion of the deterministic model into an econometric one may involve not only suitably specified error terms, but also temporally or spatially lagged dependent and or independent variables. The 'spatial' econometric developments in this general area represent a research frontier, and have been extensively reviewed and developed in Anselin (1988, 1992a, b).

Finally, let us note that in the more recent econometrics, the concept of 'data generating process' (Spanos, 1986; Darnell and Evans, 1990; Davidson and MacKinnon, 1993) has been used to justify including 'additional variables' at the stage when an econometric model is constructed from a deterministic one. Such additional variables are not part of the theoretical deterministic model, but are required by the data generating process which produced the data in which the theoretical relationship under consideration is embedded. All the developments touched upon here are potentially relevant to the construction of econometric catastrophe models.

It is important to point out that every one of the types of catastrophe models and structures discussed here can be converted into stochastic models. Specifically, models and mathematical structures of the gradient or potential types, canonical or non-canonical, expanded or nonexpanded, of catastrophes elementary or otherwise, can be converted into statistical or econometric models.

\section{A DEMONSTRATION}

The catastrophe models prevalent in the earlier catastrophe literature are deterministic, unexpanded, and are often based on canonical structures. These types of models tended to be associated with an abstract and oversimplified substantive modeling, based on variables unrelated to empirical referents and on relationships inadequately anchored to the causative presuppositions and mechanisms that are so prominent in substantive literatures. Possibly, the future prospects of CT's applications rest on the types of models that are better suited to fit within established substantive analytical literatures. These models are more likely to be based on generalized canonical or non-canonical catastrophe structures, and to be expanded and stochastic.

The demonstration that follows centers on the construction and estimation of an expanded econometric cusp catastrophe model of modern economic growth. It involves the modeling of economic growth over the 1700-1910 time span. The earlier portion of this time horizon was still characterized by a premodern dynamic. In premodern times the product per capita grew very slowly. With the industrial revolution, in the countries that experienced it, the product per capita went through a phase of accelerated 'explosive' growth, later followed by retardation. The question is: how, and on the basis of which reasoning, can we formulate a single mathematical model capable of representing these behaviors?

In the sections that follow, first the aspects of the cusp catastrophe that are relevant to the modeling of modern economic growth are brought 
into focus. Then an econometric expanded cusp catastrophe model of modern economic growth is constructed, estimated, and evaluated.

\section{The Cusp Catastrophe}

The canonical cusp catastrophe equations involve one state variable and two control parameters. Depending upon the values of the control parameters, the 'topology' of the system defined by these equations is characterized by one or two stable equilibria. The smooth change of the control parameters across critical thresholds can bring about the transition of the system from a 'low' stable equilibrium, to two stable equilibria, and again to a single 'high' stable equilibrium. As noted earlier, there are two equivalent canonical equations of the cusp catastrophe (and in general of all 'elementary' catastrophes), one in terms of a 'potential' function, and the second in the form of a gradient differential equation. The gradient equation is the one dealt with here.

In the gradient canonical equation of the cusp catastrophe (3) $x$ is the state variable, and $u, v$ are control parameters. In Zeeman's terminology $u$ is a 'splitting factor' and $v$ is a 'normal factor'. The parameter $u$ determines whether the system has one or can have two stable equilibria. When $u>0$ only one stable equilibrium can exist whatever the value of $v$. When $u<0$ it depends upon the values of $v$ whether the system has a single low level stable equilibrium, or a low level and a high level stable equilibria, or a single high level equilibrium. Suppose that $v=0$ and that $u$ changes from a positive value to a negative one. At $u=0$ the stable equilibrium that exists for $u>0$ bifurcates into a low and an high stable equilibria.

The equilibria of (3) are the values of $x$ for which $\dot{x}=0$, namely for which

$$
x^{3}+u x+v=0 .
$$

The values of $x$ in correspondence to which $\dot{x}$ attains a local maximum or minimum satisfy the condition

$$
3 x^{2}+u=0 .
$$

The set of values of $x$ that satisfy simultaneously (14) and (15) denote those equilibrium $x^{\prime}$ s at which the extrema of $\dot{x}(x)$ touch the zero axis. Eliminating $x$ from (14) and (15) yields the cusp curve

$$
4 u^{3}=27 v^{2}
$$

A switch in topology takes place at the values of $u$ and $v$ satisfying (16), that constitute the 'catastrophe set'.

The canonical cusp equation (3) can be, and has been used for modeling substantive phenomena, but in many circumstances it is preferable to employ the more flexible generalized canonical cusp structure (8) that adjusts better to substantive variables and data.

By 'comparing' Eq. (8) and (11) the $\alpha$ 's in (8) can be related to the structural parameters $u, v$, $h$, and $w$, as follows:

$$
\begin{gathered}
\alpha_{3}=h, \\
\alpha_{2}=3 h w, \\
\alpha_{1}=h\left(w^{2}+u\right), \\
\alpha_{0}=h\left(w^{3}+u w+v\right) .
\end{gathered}
$$

If the $\alpha$ 's are given, from (17) through (20), we can easily obtain the linkage parameters $h$ and $w$ and the control parameters $u$ and $v$. Namely

$$
\begin{gathered}
h=\alpha_{3}, \\
w=\alpha_{2} / 3 \alpha_{3}, \\
u=\left(\alpha_{1} / \alpha_{3}\right)-3\left(\alpha_{2} / 3 \alpha_{3}\right)^{2}, \\
v=\left(\alpha_{0} / \alpha_{3}\right)-\left(\alpha_{2} / 3 \alpha_{3}\right)\left(\left(\alpha_{1} / \alpha_{3}\right)-2\left(\alpha_{2} / 3 \alpha_{3}\right)^{2}\right) .
\end{gathered}
$$

Thus, if the structural parameters $h, w, u, v$ are given the $\alpha$ 's can be obtained; and if the $\alpha$ 's are 
given the structural catastrophe parameters can be obtained.

Suppose that (8) is converted into an econometric model by adding to its right-hand side an error term $\epsilon$ :

$$
\dot{z}=\alpha_{3} z^{3}+\alpha_{2} z^{2}+\alpha_{1} z+\alpha_{0}+\epsilon .
$$

We can then estimate the $\alpha$ 's using empirical data, and then using (21)-(24) obtain estimates of the structural catastrophe parameters.

The unexpanded econometric model (25) is useful, but limited in scope. It can only establish whether for given empirical data one or two stable equilibria occur, and thus it opens the way to 'comparative statics' types of analyses.

If some or all of the $\alpha$ 's in (25) are expanded into variables indexing some suitable substantive context, the terminal model obtained can be used to implement comparative statics analyses. Upon estimation, one such terminal model will yield estimates of the $\alpha^{\prime}$ s at each point in expansion space, and consequently it allows also estimating the structural catastrophe parameters at each point in expansion space.

If we wish instead to establish whether a catastrophic switch across dynamics has occurred the $\alpha^{\prime}$ s of (25) should be expanded into deterministic or stochastic functions of time. To exemplify, let us expand the parameters of (25) into linear stochastic functions of time $t$ :

$$
\alpha_{i}=\lambda_{i 0}+\lambda_{i 1} t+\eta_{i}
$$

where $\eta_{\mathrm{i}}$ is a $\mathrm{RV}$ associated with the $i$ th expansion equation.

The terminal model obtained by replacing the right-hand sides of (26) into (25) is

$$
\begin{aligned}
\dot{z}= & \left(\lambda_{30}+\lambda_{31} t\right) z^{3}+\left(\lambda_{20}+\lambda_{21} t\right) z^{2} \\
& +\left(\lambda_{10}+\lambda_{11} t\right) z+\left(\lambda_{00}+\lambda_{01} t\right)+m,
\end{aligned}
$$

where $m=\eta_{3} z^{3}+\eta_{2} z^{2}+\eta_{1} z+\eta_{0}+\epsilon$.

Upon estimation (27) can establish whether over time the systemic equilibria changed in value, and whether a switch across topologies occurred when.

In closing on this point let us note that the parameters of (27) could be also expanded into variables indexing a substantive context to produce a terminal model suited for a 'comparative dynamics' analysis. Such model could establish, for instance, whether changes in dynamics occurred when and where across the substantive context considered.

Let us now consider why and how these concepts and related mathematical structures can be applied to the modeling of modern economic growth. Specifically, let us discuss briefly 'modern economic growth', then bring into focus why the cusp catastrophe notions can give a useful insight into its dynamic, and articulate how a cusp catastrophe model of modern economic growth can be arrived at.

\section{A Cusp Model of Modern Economic Growth}

The dynamics of the product per capita, $y$, for the countries of North-West Europe over the 1700-1910 time horizon was characterized by the following. Before the industrial revolution, that started circa in 1750 in the UK, the product per capita was stagnant at premodern low values. It has been noted that in premodern times the rate of growth of $y$ was so small to be negligible over any short to medium time interval. Instead, the industrial revolution brought about a phase of accelerated growth of product per capita, that was eventually followed by a phase of retardation (Kuznets, 1966; 1967; 1971). Economic growth theory has been a leading theme in modern economics (Hamberg, 1971; Burmeister and Dobell, 1970; Wan, 1971; Barro and Sala-I-Martin, 1995).

The premodern stagnation in product per capita has been theorized as the results of a Malthusian trap (Boulding, 1955). The explosive growth of $y$ at the onset of the industrial revolution has been the focus of extensive theoretical and historical literatures (Nelson, 1965; Leibenstein, 1957; Rostow, 1960; Kuznets, 1971). The subsequent retardation in the growth rates of product per 
capita has also been the object of theories and data analyses reviewed in Casetti (1986) and of the more recent literatures on the so-called 'convergence' (Baumol et al., 1994).

Here we are concerned with the more formal aspects of the dynamics of the product per capita, rather than on the economic and social mechanisms suggested to explain it. Within this perspective, the premodern stagnation of $y$ can be conceptualized as the result of a slowly moving low level 'point attractor'. A country's subsequent explosive economic growth can be then construed as the initial effect of its capture by a high level point attractor possibly resulting from the disappearance of its low level counterpart. And finally the retardation in economic growth can be also explained by an increasing closeness to the high level attractor, that is also in the process of increasing slowly.

The empirical analyses to follow are based on (27), which is an econometric gradient generalized-canonical cusp equation with all its parameters expanded with respect to time. This equation, for $\dot{z}$ and $z$ denoting, respectively, percentage rate of change and logarithm of GNP per capita, is well suited to test whether the hypothesized switch in topology and temporal changes in stable equilibria did occur for the countries and over the time horizon considered.

The analyses are based on the GNPs per capita for the UK, Denmark, Sweden, and Norway, for the years $1830,1840, \ldots, 1910$, published in Bairoch (1976, Table 6, p. 286). These data are in 1960 US dollars and are based on three year averages. Annual percentage growth rates of GNP per capita for the decades 1830-1840 to 1900-1910, and GNP per capita at the midpoints of these decades were calculated using these data. The countries included in the sample were selected because they are close enough to each other geographically and otherwise. Time $t$ is in deviation from the year 1800 .

The time interval 1700-1910, addressed in the analysis, however, is wider than the data coverage. The available data extend over the explosive growth phase and over a portion of the growth retardation phase, and ends before the period of convulsions and dislocations from World War 1 to the early 1950s. However, the data available begin with 1830 . Thus the data leave the crucial premodern stagnation uncovered.

In order to remedy this substantial shortcoming of the data 'prior information' has to be entered into the analysis. This could be accomplished by Mixed Estimation, or by Bayesian regression. The approach followed here was based on a 'quick and dirty' constrained regression, that represents a limiting case of Mixed Estimation and can be justified by a sufficiently strong confidence in the prior information. Specifically, the estimation of (27) was carried out subject to the condition that in the year 1700 the product per capita of the countries in the sample was 100 US $1960 \$$ per capita, and its rate of growth was zero.

A description of the specification search carried out to parametrize Eq. (27) is of no interest here. It will suffice to say that it produced the following estimated equation:

$$
\begin{gathered}
\dot{z}=246.328-133.54 z+0.002199 z t \\
(3.38) \quad(-3.47) \quad(2.54) \\
+24.0247 z^{2}-1.43191 z^{3} \\
(3.55) \quad(-3.62)
\end{gathered}
$$

The $t$ values are shown in parentheses under their respective regression coefficients. The equation is associated with an $R$-square of 0.472 and an adjusted $R$-square of 0.415 .

The evaluation of this estimated equation centers on determining whether it is consistent with the notion that the dynamics of product per capita for the countries and time horizon selected involved a cusp catastrophe. The first step consisted in plotting the $\dot{z}(y)$ relationship it implies at a sequence of points in time, and specifically, for the years 1725, 1750, 1775, and 1800. For the sake of clarity, let us be reminded that the $\dot{z}(y)$ relationship is between the percentage rate of change of product per capita, $\dot{z}$, and the product per capita, $y$. The plots are given in Fig. 1 . 


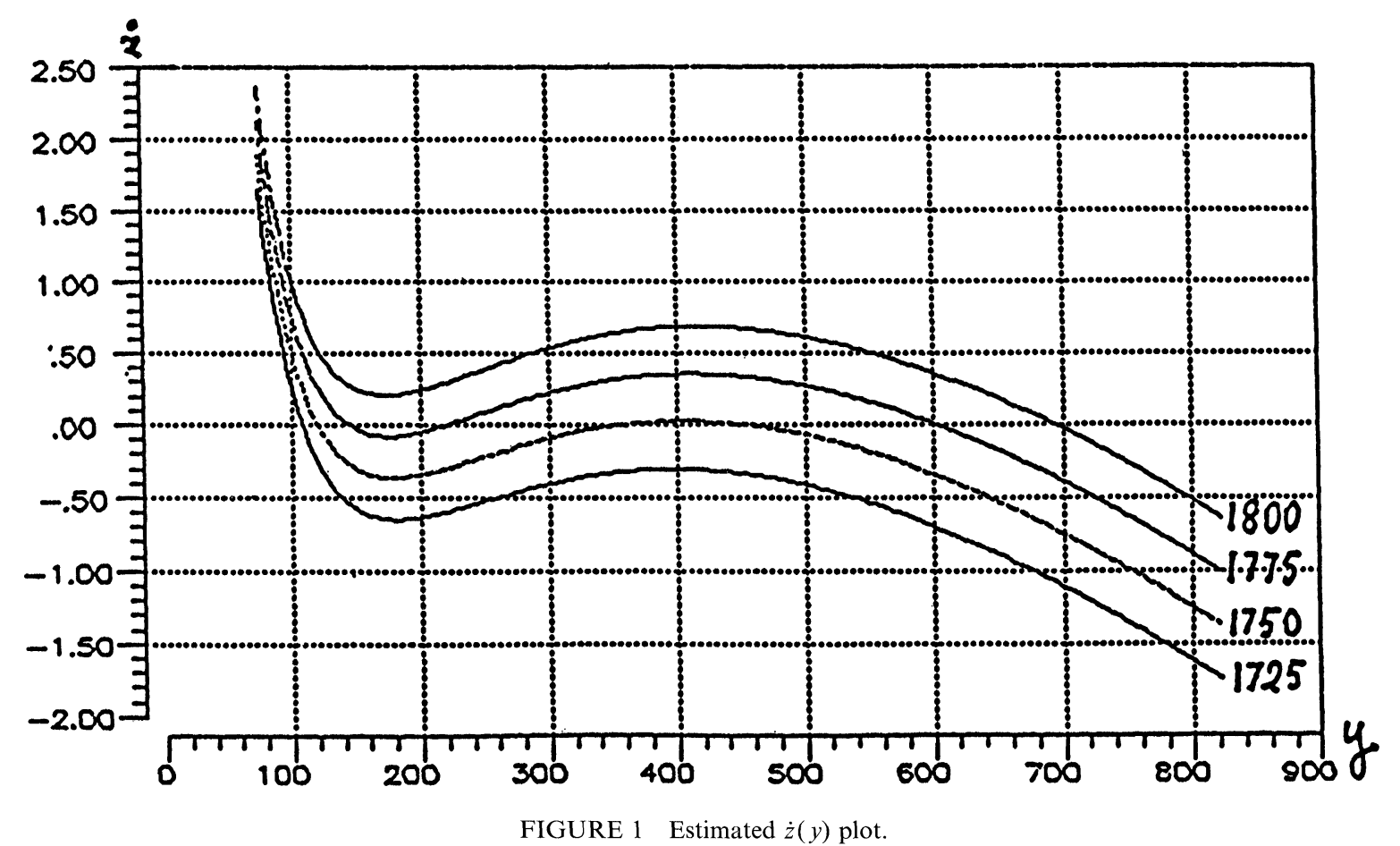

Figure 1 shows that between 1725 and 1800 a change in topology did occur. The 1725 curve intersects the zero axis in one point only, thus indicating that before the industrial revolution only a low level 'Malthusian' stable equilibrium existed. The 1750 and 1775 curves show three equilibria: a low level stable equilibrium, a high level stable equilibrium, and an unstable equilibrium between them. Finally, the 1800 curve is characterized by a single stable high level equilibrium.

The second evaluation of the estimated equations is in terms of estimated structural parameters. The regressions are based on substantive variables and parameters. The structural coefficients and variables are the control parameters and state variable that appear in CT, plus the $h$ and $w$ parameters that link the CT to the substantive variables. There is a substantial advantage to be gained by obtaining estimated structural parameters: in this manner we can relate different estimates within the same substantive problem, as well as estimates from altogether different analyses in the same and in other substantive areas to the common yardsticks represented by the control parameters $u$ and $v$.

The approach to obtaining estimated $u, v, h$, and $w$, that is employed here is fully general. As soon as we have estimates of the $\alpha$ 's appearing in Eq. (26), we can calculate from them the structural parameters using Eq. (21)-(24). These estimated $\alpha$ 's are obtained directly when we are dealing with unexpanded catastrophe models. Whenever instead we are dealing with expanded models, the estimated expansion equations can be used to evaluate estimated $\alpha$ 's at any point in expansion space. Then, from these we can again obtain the estimated structural parameters for that point in expansion space using Eq. (21)-(24).

In this demonstration, the expansion space is time. The estimated $\alpha$ 's were computed for the years $1700,1710, \ldots$ through 1850 , and then estimated structural parameters were obtained from them. The trajectory in control space corresponding to the estimated $u$ and $v$ is shown in Fig. 2. This trajectory shows that the cusp lines are 


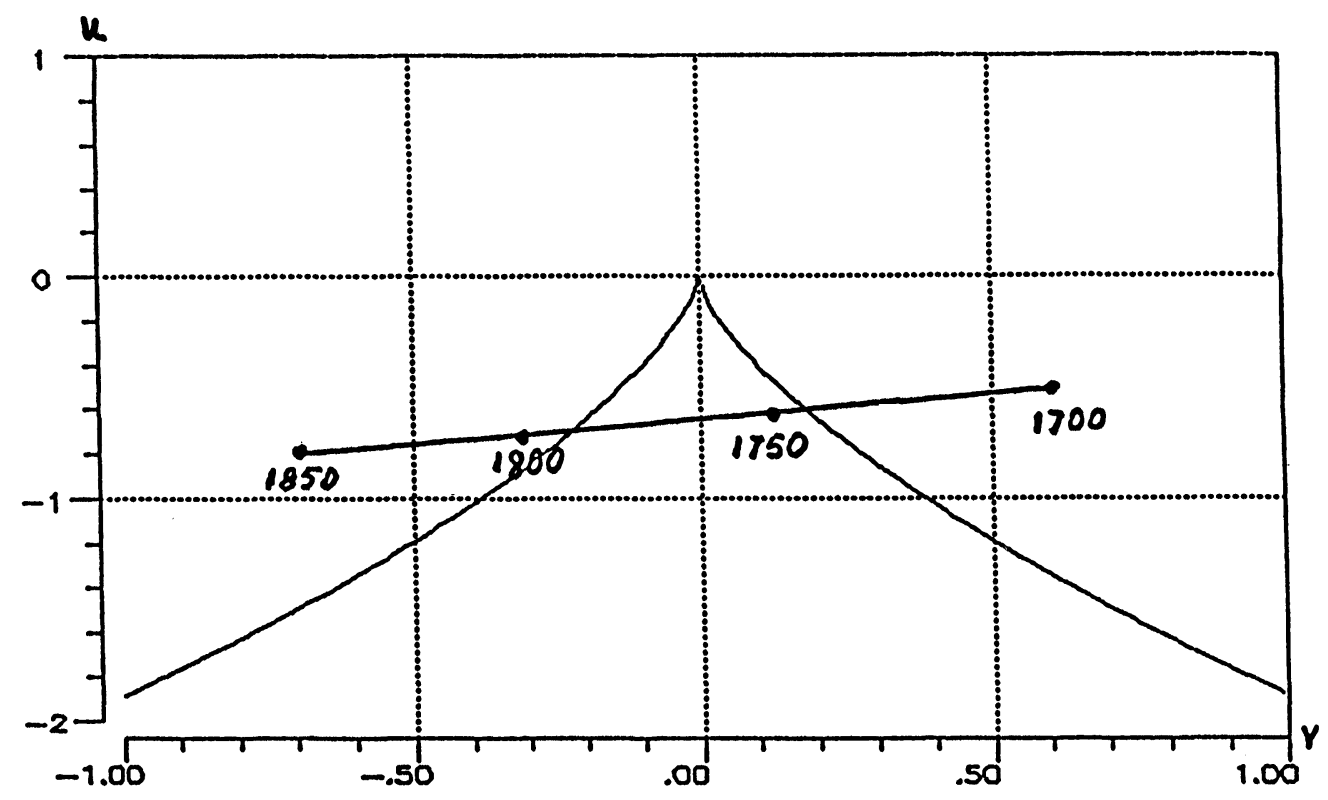

FIGURE 2 Plot of estimated $u, v$ trajectory.

crossed twice. Trajectories crossing the catastrophe set twice are the ones that produce the succession of topologies with one, then two, and then again one stable equilibria typical of the cusp catastrophe.

\section{CONCLUSION}

The themes discussed in this paper are all concerned with the application of catastrophe theory. Specifically, the paper touched upon these questions: what did CT add to our ability to construct mathematical models of realities? Why CT was received early on with an enthusiasm later followed by a wave of sharp criticisms? What are the prospects for CT's future?

The applications of mathematics in general, and in this case of CT, center on the mathematical modeling of realities. The mathematical models come into existence when analytical mathematical structures such as equations are linked to a substantive frame of reference by interpreting substantively the variables and parameters in the structures. The application of CT involves linking analytical catastrophe structures to the substantive frames of reference of substantive disciplines.

The positive response that followed the introduction of CT was due to its having generated a widespread awareness of the discontinuities brought about by the smooth change of control parameters across critical threshold, and by having pinpointed well-defined catastrophe types, some of which proved very useful. However, the catastrophy models based on canonical formulations prevalent in earlier applications were often imperfectly suited to the practices of the substantive scholars, especially in the social sciences. This mismatch contributed to the critical appraisals of CT. In this paper it is argued that non-canonical, expanded, stochastic catastrophe models and structures hold considerable promise with respect to the application of CT. The themes of this paper were demonstrated by constructing an econometric expanded gradient generalizedcanonical cusp model of modern economic growth, and then by estimating it and evaluating its performance. 


\section{Acknowledgement}

Comments and criticisms by Professors S.J. Guastello, Marquette University, and M. Sonis, Bar-Ilan University, are gratefully acknowledged.

\section{References}

Anselin, L., Spatial Econometrics: Methods and Models, Kluwer Academic, Dordrecht, 1988.

Anselin, L., "Spatial dependence and spatial heterogeneity: model specification issues in the spatial expansion paradigm", in Applications of the Expansion Method, J.P. Jones III and E. Casetti (eds), Routledge, London and New York, 1992a, pp. 334-354.

Anselin, L., Spacestat Tutorial, NCGIA, Technical Software Series S-92-1, November 1992b.

Arnol'd, V.I., Catastrophe Theory, 3rd edition, Springer-Verlag, Berlin, 1992.

Bairoch, P., "Europe's Gross National Product: 1800-1975", Journal of European Economic History 5, 1976, 273-340.

Barro, R.J. and Sala-I-Martin, X., Economic Growth, McGraw-Hill, New York, 1995.

Baumol, W.J., Nelson, R.R. and Wolff, E.N. (eds), Convergence of Productivity, Cross-National Studies and Historical Evidence, Oxford University Press, Oxford, 1994.

Boulding, K.E., "The Malthusian model as a general system", Social and Economic Studies 4(3), September 1955, 195205.

Burmeister, E. and Dobell, A.R., Mathematical Theories of Economic Growth, The Macmillan Co. Collier-Macmillan Ltd., London, 1970.

Brown, C., Chaos and Catastrophe Theories, Sage, 1995.

Casetti, E., "Generating models by the expansion method: applications to geographic research", Geographical Analysis 4, 1972, 81-91.

Casetti, E., "The dual expansions method: an application to evaluating the effects of population growth on development", IEEE Transactions on Systems, Man, and Cybernetics SMC-16(1), January-February 1986, 29-39.

Casetti, E., "Testing catastrophe hypotheses; expanded differential equations and the dynamic of urbanization", SocioSpatial Dynamics 2, 1991, 65-79.

Casetti, E., "The expansion method, mathematical modeling, and spatial econometrics", forthcoming in a special issue on spatial Econometric, L. Anselin and S. Rey (eds), of the International Regional Science Review, 1997.

Cobb, L., "Stochastic catastrophe models and multimodal distributions", Behavioral Science 23, 1978, 360-374.

Cobb, L., Koppstein, P. and Chen, N.H., "Estimation and moment recursion relations for multimodal distributions of the exponential family", Journal of the American Statistical Association 78, 1983, 124-130.

Cobb, L. and Zacks, S., "Applications of catastrophe theory for statistical modeling in the biosciences", Journal of the American Statistical Association 80, 1985, 793-802.

Cobb, L., "Statistical catastrophe theory", in Encyclopedia of Statistical Sciences, Kotz S. and Johnson N.L. (eds), Vol. 8, Wiley, New York, 1992, pp. 634-640.

Darnell, A.C. and Evans, J.L., The Limits of Econometrics, Elgar, Hants UK, 1990.
Davidson, R. and MacKinnon, J.G., Estimation and Inference in Econometrics, Oxford University Press, New York, Oxford, 1993.

Dendrinos, D.S., "Volterra-Lotka ecological dynamics, gravitational interaction, and turbulent transportation: an integration", Sistemi Urbani 2, 1989, 203-216.

Dendrinos, D.S. and Mullally, H., "Fast and slow equations: the development patterns of urban settings", Environment and Planning A 13, 1981, 819-827.

Dendrinos, D.S. and Mullally, H., Urban Evolution, Studies in the Mathematical Ecology of Cities, Oxford University Press, London, 1985.

Dendrinos, D.S. and Sonis, M., Chaos and Socio-Spatial Dynamics, Springer-Verlag, New York, 1990.

Drazin, P.G., Non Linear Systems, Cambridge University Press, Cambridge, 1992.

Gilmore, R., Catastrophe Theory for Scientists and Engineers, Wiley, New York, 1981.

Glendinning, P., Stability, Instability and Chaos: An Introduction to the Theory of Nonliner Differential Equations, Cambridge University Press, Cambridge, 1994.

Guastello, S.J., "Moderator regression and the cusp catastrophe: application of two-stage personnel selection, training, therapy and policy evaluation", Behavioral Science 27, $1982,259-272$.

Guastello, S.J., "A butterfly catastrophe model of motivation in organizations: academic performance", Journal of Applied Psychology 72, 1987, 165-182.

Guastello, S.J., "Catastrophe Modeling of the Accident Process: Organizational Subunit Size", Psychological Bulletin 103, 1988, 246-255.

Guastello, S.J., Chaos, Catastrophe, and Human Affairs: Application of Non Linear Dynamics to Work, Organizations, and Social Evolution, Lawrence Erlbaum Associates Publishers, Mahawah, New Jersey, 1995.

Hale, J. and Kocak, H., Dynamics and Bifurcations, SpringerVerlag, New York, 1991.

Haken, H., Synergetics, Non-Equilibrium Phase Transitions and Social Measurement, 3rd edition, Springer-Verlag, Berlin, 1983.

Hamberg, D., Models of Economic Growth, Harper \& Row, New York, 1971.

Johnston, J., Econometric Methods, McGraw-Hill, New York, 1963.

Kuznets, S., Modern Economic Growth, Rate Structure and Spread, Yale University Press, New Haven and London, 1966.

Kuznets, S., "Population and economic growth", Proceedings of the American Philosophical Society, Vol. 111, 1967, pp. 170-193.

Kuznets, S., Economic Growth of Nations: Total Output and Production Structure, Harvard University Press, Cambridge, Massachusetts, 1971.

Leibenstein, H., Economic Backwardness and Economic Growth, Wiley, New York, 1957.

Lung, Y., "Complexity and spatial dynamic modeling. From catastrophe theory to self-organizing process: a review of the literature", Annals of Regional Science 22(2), July 1988, $81-111$

Nelson, R.R., "A theory of the low level equilibrium trap in underdeveloped economies", American Economic Review 46, 1965, 896-908.

Nelson, R.R., "Growth models and the escape from the lowlevel equilibrium trap: the case of Japan", Economic Development and Cultural Change 8(4), July 1960, 378-388. 
Oshima, H.T., "Economic growth and the "critical minimum effort", Economic Development and Cultural Change 7(4), July $1959,467-478$.

Poston, T. and Stewart, I., Catastrophe Theory and its Applications, Pitman, London, San Francisco, Melbourne, 1978.

Rosser, B.J., From Catastrophe to Chaos: A General Theory of Economic Discontinuities, Kluwer Academic Publishers, Norwell, 1991.

Rostow, W.W., The Stages of Economic Growth: A NonCommunist Manifesto, Cambridge University Press, Cambridge, England, 1960.

Spanos, A., Statistical Foundations of Econometric Modeling, Cambridge University Press, Cambridge, 1986.

Sussmann, H.J. and Zahler, R.S., "Catastrophe theory as applied to the social and biological sciences: a critique", Synthese, 37 1978, 117-216.

Thom, R., Structural Stability and Morphogenesis, Benjamin, Reading, MA, 1975.

Tu, P.N.V., Dynamical Systems, Springer-Verlag, Berlin, Heidelberg, 1992.
Wan, H.Y.Jr., Economic Growth, Harcourt Brace Jovanovich Inc., New York, Chicago, San Francisco, Atlanta, 1971.

Wilson, A.G., Catastrophe Theory and Bifurcation, Application to Urban and Regional Systems, Croom Helm, London, 1981.

Wilson, A.G. and Kirby, M.J., Mathematics for Geographers and Planners, 2nd edition, Clarendon Press, Oxford, 1980.

Zahler, R.S. and Sussmann, H.J., "Claims and accomplishments of applied catastrophe theory", Nature 269(27), October 1977, 759-763.

Zeeman, E.C., "Differential equations for the heartbeat and nerve impulse", in Toward a Theoretical Biology, Waddington C.H. (ed), Aldine-Atherton, Chicago, IL, 1972.

Zeeman, E.C., "Differential equations for the heart and nerve impulse", in Dynamical Systems, Peixoto (ed), Academic Press, New York, 1973.

Zeeman, E.C., Catastrophe Theory, Addison-Wesley, Reading, MA, 1977. 


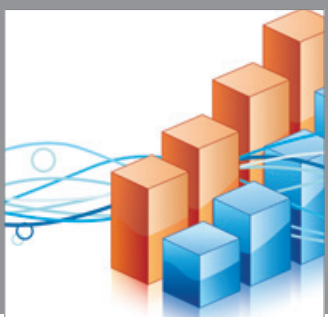

Advances in

Operations Research

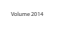

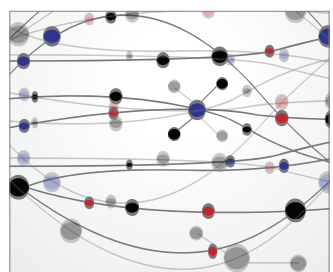

\section{The Scientific} World Journal
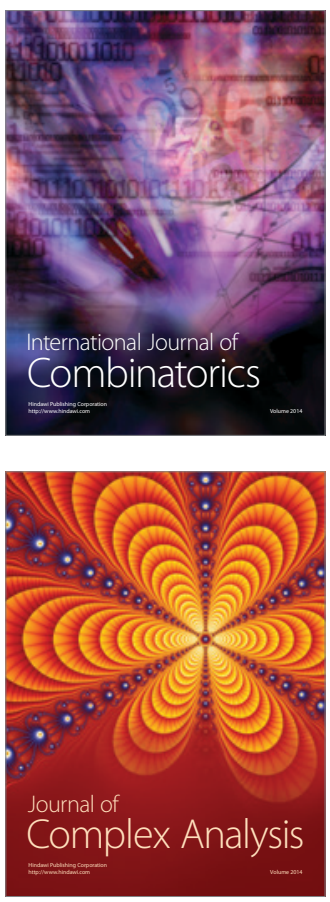

International Journal of

Mathematics and

Mathematical

Sciences
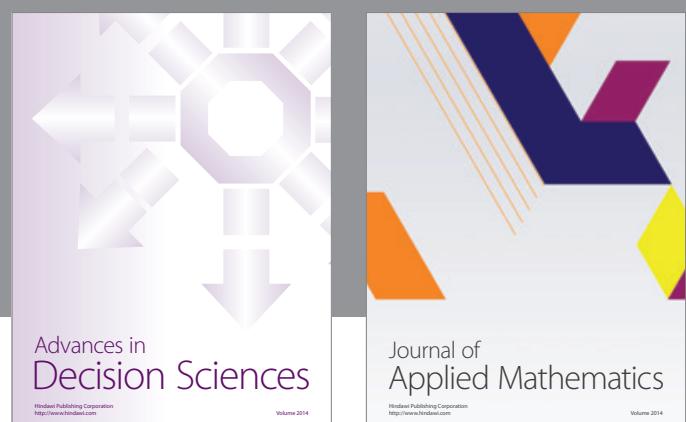

Journal of

Applied Mathematics
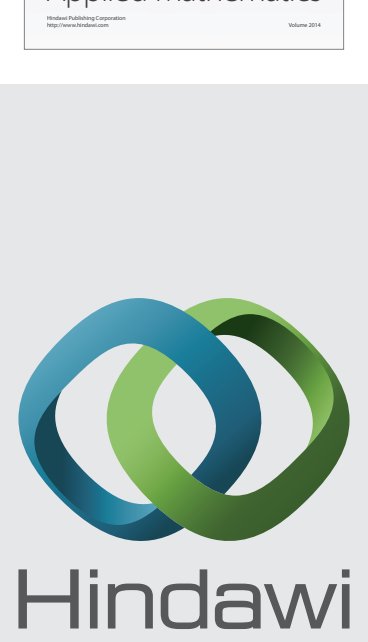

Submit your manuscripts at http://www.hindawi.com
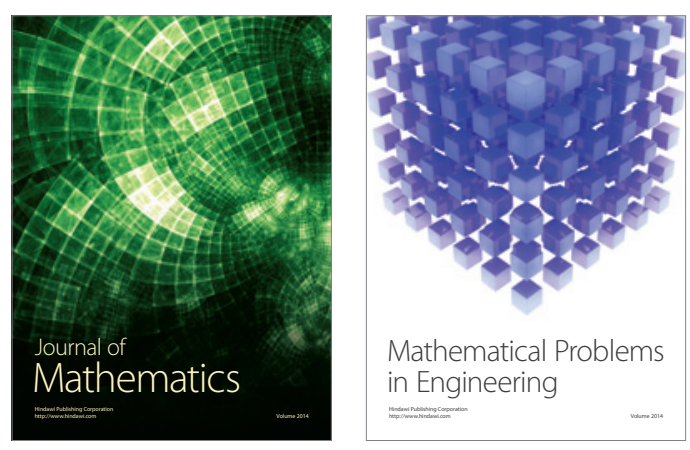

Mathematical Problems in Engineering
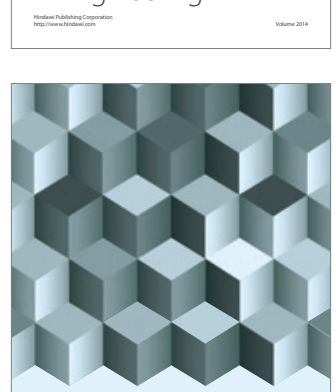

Journal of

Function Spaces
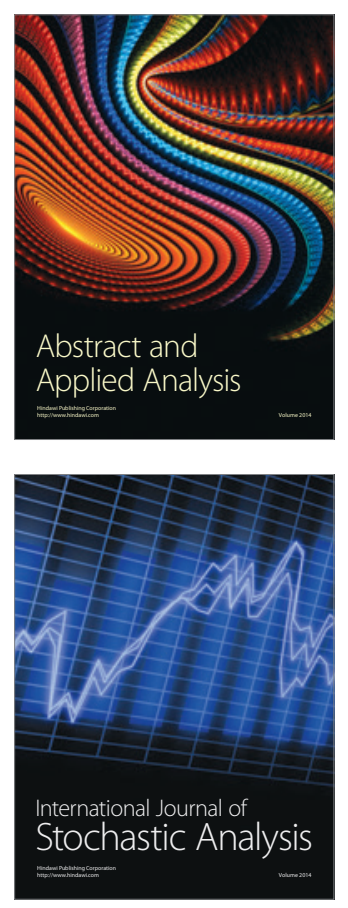

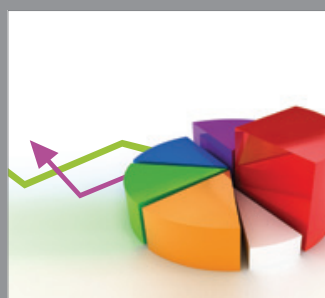

ournal of

Probability and Statistics

Promensencen
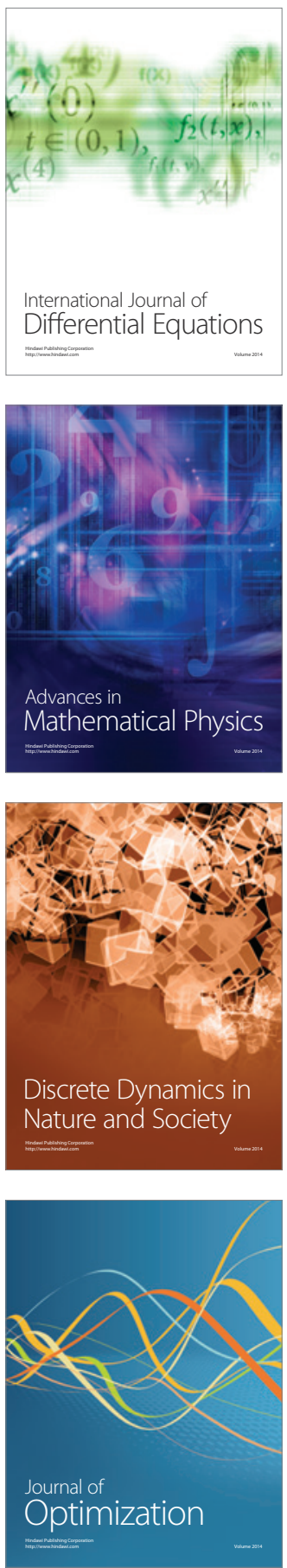